\title{
Phenotypic and Genotypic Characters of Mycobacteria Isolated from Extrapulmonary Tuberculosis Patients in Sohag Governorate
}

\author{
MostafaYousef El-Mishad, AbeerSheneef Mohamed, Tamer Mohamed \\ Mahmoud, EkramAbd-El Rahman Mahmoud
}

Medical Microbiology \& Immunology Department, El-Azhar University and Sohag University

\begin{abstract}
Tuberculosis is a major global health problem. ExtrapulmonaryTB(EPTB) refers to TB involving organs other than the lungs. It shoulddrag more attention after increasing numbers of immunocompromised individualswith higher risk of developing EPTB.

Aim: This study aimed to detectEPTB in clinically suspected cases and identificationof mycobacteria species by phenotypic and genotypicmethods.

Materials and Methods: A total of 100 clinical specimens were collected during the study period from September 2016 to January 2018 at Sohag University from patients suspected to have EPTB. All samples subjected to ZN staining \& culture on LJ media then all positive culture were subjected for identification of mycobacteria speciesby two methods, phenotypically by biochemical tests and genotypically by conventional PCR for detection of mycobacterium Tuberculosis Complex ( MTC).Immunochromatographic tests were done for all strains with negative PCR in addition to some strains with positive PCR for confirmation and for comparison between immunochromatographic test and both biochemical test and PCR in identification of MTC.

Results: Mycobacteria were isolated from 66 samples of which the highest number of isolates were obtained from urine samples followed by stool samples then other extrapulmonary samples. By using different methods of identification, 53 isolates were identified as MTC and 13 isolates were identified as nontuberculus mycobacteria (NTM).

Conclusion: Our results suggest that due attention should be given for EPTB and rapid differentiation between MTC and NTM is very important for the proper management of patients with mycobacterial infections.
\end{abstract}

\section{INTRODUCTION}

Tuberculosis (TB) is a chronic, granulomatous, bacterial infection that may display multisystemic involvement. It still constitutes a major global health problem and it is estimated that almost one-third of the population is infected worldwide (Ates et al., 2015).

Tuberculosis is caused by Mycobacterium tuberculosis complex (MTC), comprises the closely related organisms as $M$. tuberculosis, $M$. africanum, $M$. bovis, $M$. bovis (BCG), $M$. microti, etc. (Halse et al., 2011).
Mycobacterium tuberculosisisthe main causative agent in the vast majority of cases .Mycobacterium bovishas a higher proportion in patients with exclusively EPTB. It accountsfor approximately 0.5$1.5 \%$ of the entire human TB cases in some developedCountries but it has a higher prevalence of infection in developingcountries (5-10\%), which has been attributed to poorbovine tuberculosiscontrol in these countries\&transmission can occur through consumption of contaminated 
milk and closecontact with infected cattle (Silaigwanaet al.,2012).

The two types of clinical manifestation of tuberculosis (TB) are pulmonary $\mathrm{TB}$ (PTB) and extrapulmonary TB (EPTB). Pulmonary TB is more common as it is a highly contagious infection (Sreeramareddy et al., 2008) whileextrapulmonary TB refers to TB involving organs other than the lungs (e.g., pleura, lymph nodes, abdomen, genitourinary tract, skin, joints, bones or meninges) (WHO, 2017).

The term "nontuberculous mycobacteria" (NTM) generally refers to mycobacteria other than the Mycobacterium tuberculosis complex and M. leprae(Griffith et al., 2007).

Several species of NTM can cause extrapulmonary diseases such as skin/soft tissue infections, lymphatic, disseminated, and nosocomial infections after surgery of varyingseverity in both sporadic and epidemic form (Maurya et al.,2015).

\section{MATERIALS AND METHODS}

The present study is a descriptive study conducted from September 2016 to January 2018 at Medial Microbiology \& Immunology Department, Sohag faculty of Medicine. The study included patients recruited from different clinical departments at Sohag University hospital and private clinics at Sohag Governorate who suspected by clinician to have TB outside the lung (EPTB). The study was approved by Institutional Ethics Committee and informed consents were obtained from all the patients. All basic demographic and clinical informations were recorded including age, sex, causes of seeking medical advice, information of potential risk factors as diabetes mellitus, hypertension, drinking unpasteurized milk and its derivative products etc.
Collection of samples: One hundred clinical specimens were collected during the study period and screened for mycobacteria species. These include 45 urine samples, 35 stool samples and 20 other samples (rather than urine \&stool) include pleural fluid samples (5), ascitic fluid samples (3), biopsies from skin lesions (3), pus from chest abscess (2), cervical lymph nodes biopsy (2), pus from breast abscess (2), biopsy from laryngeal mass (1), biopsy from vocal cord thickening (1) and swab from skin sinus (1).

Detection of mycobacteria: (i) ZiehlNeelsen (ZN) staining : Mycobacteria appeared under light microscope as thin pink rods (sometimes appeared short \& beaded as in urine) arranged singly or in small groups on blue background. (ii) Culture on LJ media:At first decontamination and concentration method was done for contaminated samples by using Petroffmethod ( $\mathrm{Na} \mathrm{OH}$ method). After that sterility test was done for each sample by inoculation on three agar plates (nutrient agar, blood agar and MacConkey) in addition to L.J media and incubate for 3 days. Mycobacterium tuberculosis complex colonies on $\mathrm{LJ}$ media are characteristically dry with a rough texture and a creamy color. Conversely, M. bovis colonies are flat and smooth. Several Mycobacterium species also produce pigments that can range from yellow to orange color.

Tests for identification of mycobacteria species: (i) Phenotypic identification A) biochemical tests include PNB (Para nitro benzoic) acid test and TCH (thiophene-2carboxylic acid hydrazide)test, nitrate reduction test strip and niacin detection kit.Results of biochemical tests shown in table (1) B)Immunochromatographic identification test (SD BIOLINE TB Ag MPT64 Rapid test, Germany). when both 
test band ( $\mathrm{T}$ ) and control band (C) appeared as colored bands this mean that the tested strain was identified as M.tuberculosis complexwhilethe presence of only control band (c) indicate that the tested strain was not identified as M.tuberculosis complex this mean it was NTM (ii) Genotypic identification of $M$. tuberculosis complex by PCR. DNA extraction was done by using DNA,RNA and protein purification kits (MACHEREY - NAGEL,Germany). The used primers were specific for

Table (1) Biochemical tests of both MTC and NTM (according to Center Lab. Of TB of Egypt)

\begin{tabular}{|l|l|l|l|l|}
\hline Potential pathogen & PNB & TCH & Niacin & Nitrate reduction test \\
\hline MTC & S & R/S & + & + \\
\hline NTM & R & R & - & $+/-$ \\
\hline
\end{tabular}

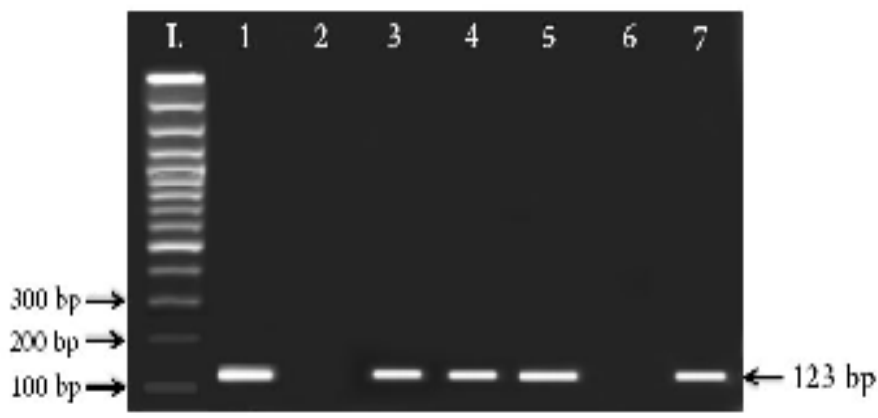

Photo (1) Agarose gel electrophoresis of amplified an insertion sequence IS6110 ( 123-bp). of $M$. tuberculosis complex

- Lane (L): fragments of (100-1000 bp) ladder

- Lane (3-4-5-7): amplified fragments of 123 bpof an insertion sequence IS6110 from different $M$. tuberculosis complex strains extracted from culture

- Lan (1):A positive control sample strain was included

- Lan (2): A negative control 


\section{RESULTS}

Out of 100 samples analyzed, mycobacteria were detected in 66 samples. The age of patient with mycobacterial infection among those positive cases was ranging between 8 years and 80 years with mean \pm S.D $(45.8 \pm 17.33)$. The highest incidence occur in age group (41-50 years old) in percent $22.7 \%$, followed by $21.2 \%$ in age group (31-40 years old) , $15.2 \%$ in age group (51-60 years old), $13.6 \%$ in age group $(61-70$ years old), $10.6 \%$ in age group (71-80 years old), $7.6 \%$ in age group (21-30 years old), 6.06\% in age group (11-20 years old) then $3.04 \%$ in age group (0-10 years old). Mycobacterial infections were more among males $(69.7 \%)$ than females $(30.3 \%)$. patients with mycobacterial infection may be presented by more thanmore than one risk factor at the same time and more than one symptom at the same time.The risk factors associated with mycobacterial infection was shown in figure (1).Urinary symptoms associated with mycobacterial infection were shown in table (2).Abdominal symptoms associated with mycobacterial infection was shown in table (3)

During our study period, all positive mycobacerialisolates (66) were tested by both biochemical tests and PCR (the gold standard test) for identification of MTC. Fifty six isolates were identified as MTC and 10 isolates as NTM by biochemical tests, whereas 53 isolates were identified as MTC and 13 isolates as NTM by PCR. So sensitivity, specificity, positive predictive value (PPV), negative predicative value (NPV) of biochemical tests in comparison with PCR method were $100 \%, 76.9 \%, 94.6 \%$ and 100respectively.

The thirteen negative strains by PCR (10 strains were identified biochemically as MOTT and 3 strains were identified biochemically as MTBC) in addition to 10 positive strains by PCR (identified biochemically as MTBC) were confirmed by immunochromatography test \& the test gave the same result of PCR.

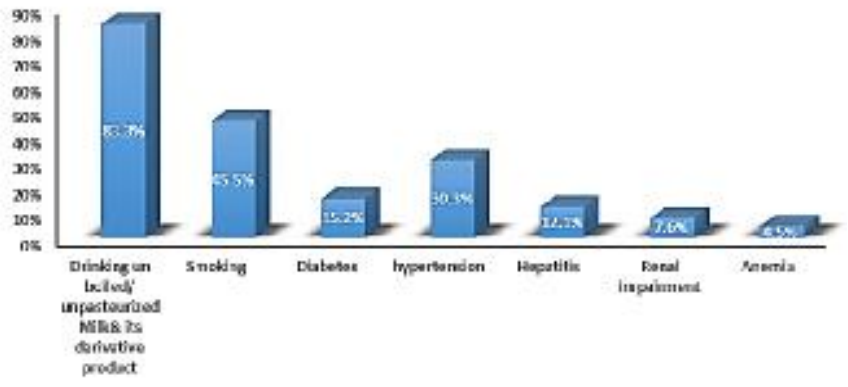

Figure (1): Risk factors for patients with mycobacteria $\left(\mathbf{N}_{.}=66\right)$

Table (2): Urinary symptoms associated with mycobacterial infection (N. =27)

\begin{tabular}{|c|c|}
\hline Urinary symptoms & No. $(\%)$ \\
\hline Frequency & $\mathbf{2 3}(\mathbf{8 5 . 2 \% )}$ \\
\hline Dysuria & $\mathbf{2 3}(\mathbf{8 5 . 2 \% )}$ \\
\hline Loin pain & $\mathbf{1 2}(\mathbf{4 4 . 4 \% )}$ \\
\hline Recurrent renal stones & $\mathbf{3 ( 1 1 . 1 \% )}$ \\
\hline Hematuria & $\mathbf{3 ( 1 1 . 1 \% )}$ \\
\hline Nocturnal enuresis & $\mathbf{5 ( 1 8 . 5 \% )}$ \\
\hline Perineal pain & $\mathbf{2 ( 7 . 4 \% )}$ \\
\hline
\end{tabular}


Table (3): Abdominal symptoms associated with mycobacterial infection $\left(\mathbf{N}_{.}=25\right)$

\begin{tabular}{|l|l|}
\hline \multicolumn{1}{|c|}{ abdominal symptoms } & No. (\%) \\
\hline Chronic diarrhea & $\mathbf{2 0}(\mathbf{8 0} \%)$ \\
\hline Chronic abdominal pain & $\mathbf{1 5}(\mathbf{6 0 \%})$ \\
\hline $\begin{array}{l}\text { Abdominal distension due to } \\
\text { Ascites }\end{array}$ & $\mathbf{3 ( 1 2 \% )}$ \\
\hline
\end{tabular}

\section{DISCUSSION}

In the present study, The age of patients with mycobacterial infection was ranging between 8 years and 80 years with Mean \pm S.D $(45.8 \pm 17.33)$ withhigh incidence above age 40 years (representing $62 \%$ of total cases). These results were in agreement with the following studies which done by Henkle et al. (2017) who reported the median age of patients with extrapulmonary isolateswas 50 years (range 0.8-92 years), a study by Peto et al. (2009) who reported The mean age of patients with EPTB was 44 years, range (0-105), a study by Umrao et al. (2016) withThe median age of NTM-positive patients in extrapulmonary samples was 48 years; the predominant age group was $>55$ years $(36.2 \%)$.On other hand, our results were different from those of Mohammadien et al. (2017)he reported that the commonest age range for extrapulmonary TB is (21-30) years and mean age is $34.8 \pm 7.1$. Also our study differ from a study done at El-Behira Governorate, Egypt on extrapulmonary cases, the cases occurred more in age groups between 15 and 29 at percent (45.5\%) (Hibah, 2015).

In our study Mycobacterial infections were more among males $(69.7 \%)$ than females $(30.3 \%)$. This finding was supported byArora and Gupta (2006), Peto et al. (2009), Chennaveerappa et al. (2011), Prakasha et al. (2013), Umrao et al. (2016). This may be due to lower notification rate for females and this This might be due to underdiagnosis or underreporting of tuberculosis in females as a result of various social and/or cultural factors, including the stigmatization of females with tuberculosis and their consequent impaired access to health care, a situation which is often seen in developing countries. On other hand, some studies showed higher incidence in female more than male these findings showed by Mohammadien et al. (2017), Noertjojo et al. (2002), Yang et al. (2004), Forssbohm et al. (2007), Sobh et al. (2016)and Henkle et al. (2017).

In our study the most prevalent risk factor in our study associated with mycobacterial infection was drinking un boiled/ unpasteurized Milk and its derivative products (representing $83.3 \%$ ), this support that the most causative organism in our study was M.bovis, which belongs to the $M$. tuberculosis complex and other members of atypical mycobacteria that transmitted by consumption of infected raw milk and its derivative products and this considered Zoonotic tuberculosis (TB). This was in accordance with De Kantor et al. (2014), WHO. (2017), Bapat et al. (2017) and Roberto et al. (2018). They reported that Zoonotic tuberculosis (TB) is a form of tuberculosis in people caused by $M$. bovis which often affects sites other than the lungs (extra- 
pulmonary). In our result smoking and diabetes also represented risk factors for mycobacterial infection in percent $45.5 \%$ and $15.2 \%$ respectively because those two factors lead to decrease in immune system of the affected person and make him more vulnerable to the mycobacterial infection this was in accordance with WHO. (2014) that reported one-third of the world's population has latent tuberculosis (TB), People infected with $M t b$ have a lifetime risk of falling ill with TB of $10 \%$. However, persons with compromised immune systems, such as people with human immunodeficiency virus (HIV), malnutrition, or diabetes mellitus or people who use tobacco, have a much higher risk of becoming ill.

Patients with mycobacterial infection in the urinary system in our study were presented by more than one symptom at the same time .In patient with mycobacterial infection in urinary system the most common symptom in our study was frequency and dysuria (each represented $85.2 \%$ ) followed by loin pain (represented $44.4 \%$ ), nocturnal enuresis(represented $18.5 \%$ ), hematuria $\&$ recurrent renal stones (each represented $11.1 \%$ ) then perineal pain (represented $7.4 \%$ ). This was near to a study done by Mohammadien et al. (2017) , he reported that the clinical presentations of the cases with genitourinary TB in Sohag was $(65 \%)$ of cases have dysuria while $(40 \%)$ have infertility. Also Huang et al. (2010) reported similar manifestation in cases with urogenital TB manifested by dysuria, frequency or urgency (53\%), fever (26\%), Flank pain (19\%), gross haematuria(19\%), scrotal nodule, pain or swelling (14\%), anorexia (7\%), while NTM infections in urogenital system presented mainly by fever (67\%) followed by Dysuria, frequency or urgency (60\%), anorexia (27\%), flank pain $(20 \%)$, scrotal nodule, pain or swelling $(20 \%)$, gross haematuria $(7 \%)$ but in our results fever and anorexia not manifested. Another study Between 1993 and 2002 support our result. It showed that patients with Urinary Tuberculosis presented mainly by frequency-dysuria $(46 \%)$ followed by flank pain (33\%), $20 \%$ of the patients were asymptomatic then macroscopic hematuria (12\%) ( Altintepe et al, 2005). Patients with mycobacterial infection in the abdomen may be presented by more than one symptom at the same time. In our study the most common symptom was chronic diarrhea (represented $80 \%$ ) followed by chronic abdominal pain (represented 60\%) then distension due to ascites (represented 12\%). This near with Mohammadien et al. (2017) study who reported that clinical presentations of the cases in TB enteritis were $(100 \%)$ with diarrhea and $(85 \%)$ with anorexia, . In TB ascites (100\%) presented with abdominal distension and $(60 \%)$ with anorexia.Also our result coincide with Sharma and Bhatia. (2004) and Kumar et al., (2003) reported that patients with . Abdominal tuberculosis have Chronic diarrhea and malabsorption in $21-75 \%$ of cases. Also Chow et al. (2002) reported the most common features of cases with Tuberculous Peritonitis were ascites (93\%), abdominal pain (73\%), and fever (58\%).On other hand Krishnaswamy et al. (2017). David et al. (1999) showed in cases of abdominal tuberculosis abdominal pain $(92 \%)$ was the most common clinical followed by anorexia $(70 \%)$ \&loss of weight $(70 \%)$. Also In a study conducted by Sharma and Bhatia. (2004) and Krishnaswamy et al. (2017), fever was recorded in half of the patients and in $40 \%$ of cases 
respectively but not manifested in our study. Also Mukewar et al. (2012), Makharia et al. (2010) and Khan et al. (2006) reported that abdominal TB had the following clinical pictures with different percent from our result, abdominal pain ,anorexia , loss of weight, fever, diarrhea, constipation and bleeding per rectum.

The difference in clinical presentation depends upon the site and type of involvement. This was supported by Rathi and Gambhire (2016) who reported that the clinical feature of TB differ according to the site and type of involvement as TB in small intestine take two forms ulcerative type (presented by diarrhea \&malabsorption) and stricturous type (presented by obstruction) and peritoneal TB also have two forms, ascitic type (presented by pain \&distension) and adhesive type (presented by obstruction), TB in large intestine have two forms, ulcerative type(presented by rectal bleeding) and hyperotrophic type (presented by lump and obstruction. TB in lymph node presented by lump obstruction.

In our study the overall positivity for mycobacterial infection in all extrapulmonary samples was $66 / 100$ $(66 \%)$. Out Of 66 isolates, $53(80.3 \%)$ were characterized as MTC and 13 (19.7\%) as NTM. The frequency of positivity for mycobacterial infection in our result was higher than those reported by both Kumari et al. (2016) and Maurya et al. (2015) which were $40 \%$ and $30.1 \%$ respectively. However incidence of MTBC and NTM among positive cases of mycobacterial infection in our result were near to those reported by both Kumari et al. (2016) in which (90\%) were characterized as MTB and (10\%) as NTM and Maurya et al. (2015) in which $(72.6 \%)$ isolates were MTC and
(27.4\%) were identified as different species of NTM.

In our studySensitivity, specificity, positive predictive value (PPV), negative predicative value (NPV) of biochemical tests in comparison with PCR method were $100 \%, \quad 76,9 \%, \quad 94.6 \% .100 \%$ respectively. These results disagreed with those results of Sadeghianet al., 2005 who reported that the sensitivity and specificity of biochemicaltests compared to PCR method were 96 and $100 \%$, respectively. Positive predicted value (PPV) and negative predicted value (NPV) were 100 and 92\%, respectively.

In our study Sensitivity, specificity, positive predictive value (PPV), negative predicative value (NPV) of biochemical test in comparison with immunochromatography were $100 \%$, $76.92 \%, 76.92 \%, 100 \%$ respectively while Sensitivity, specificity, positive predictive value (PPV), negative predicative value (NPV) of immunochromatography in comparison with PCR were $100 \%, 100 \%, 100 \%$, $100 \%$ respectively. Our findings supported by Somoskovi et al.(2002) and Tortoli et al. (2001) who reported that conventional methods like a panel of different biochemical tests are unable to distinguish more than 100 Mycobacterial species, since they overlap in their biochemical properties. Also Using the same ICT kit by Chihotaet al (2010) and Singh et al. (2012) supported our findings \& reported sensitivity and specificity of $100 \%$ for ICT test for differentiation of MTBC and NTM species.Also The SD MPT64 assay was evaluated on a large number of clinical isolates in India and performed with $100.0 \%$ sensitivity and specificity(Shenoy and Mukhopadhyay, 2014). 
In conclusion, extrapulmonary TB infection rate in Sohag governorate is high. So an early and rapid diagnosis of EPTB as well as distinction between the different members of MTC and differentiation between MTC and NTM are essential to determine the etiology of mycobacterial infections and to optimize efficient treatment.

\section{REFERENCES}

1-Altintepe, L., Tonbul, H. Z., Ozbey, I., Guney, I., Odabas, A. R., Cetinkaya, R., ...\&Selcuk, Y. (2005). Urinary tuberculosis: ten years' experience. Renal failure, 27(6), 657-661.

2-Arora, V. K., \& Gupta, R. (2006). Trends of extra-pulmonary tuberculosis under revised national tuberculosis control programme: A study from South Delhi. Indian Journal of Tuberculosis, 53(2), 77.

3-Ates GS, Bozkus F, Inci MF, Kokoglu OF, Ucmak H, Ozden S, et al (.2015) Evaluation of pulmonary and extrapulmonary tuberculosis in immunocompetent adults: a retrospective case series analysis. Med PrincPract Med PrincPract.;24(1):75-9.

4- Bapat, P. R., Dodkey, R. S., Shekhawat, S. D., Husain, A. A., Nayak, A. R., Kawle, A. P., ... \&Kashyap, R. S. (2017). Prevalence of zoonotic tuberculosis and associated risk factors in Central Indian populations. Journal of epidemiology and global health, 7(4), 277-283.

5- Chennaveerappa PK,,Siddharam, S. M., Halesha, B. R., Vittal, B. G., \&Jayashree, N. (2011). International Journal of Biological \& Medical Research. Int J Biol Med Res, 2(2), 487489.

6-Chihota, V. N., Grant, A. D., Fielding, K., Ndibongo, B., Van Zyl, A., Muirhead, D., \& Churchyard, G. J. (2010). Liquid vs. solid culture for tuberculosis: performance and cost in a resource-constrained setting. The international journal of tuberculosis and lung disease, 14(8), 1024-1031.
7-De Kantor, I. N., Torres, P. M., Roxo, E., Garin, A., ParedesNoack, L. A., Sequeira, M. D., ... \& da Silva Telles, M. A. (2014). Mycobacterium bovis infection in humans and animals with an emphasis on countries in Central and South America. Zoonotic Tuberculosis: Mycobacterium bovis and Other Pathogenic Mycobacteria, 35-49.

8-Forssbohm, M., Zwahlen, M., Loddenkemper, R., \&Rieder, H. L. (2007).Demographic characteristics of patients with extrapulmonary tuberculosis in Germany. European respiratory journal, 31:99-105.

9-Griffith, D. E., Aksamit, T., BrownElliott, B. A., Catanzaro, A., Daley, C., Gordin, F., ...\&Iseman, M. (2007). An official ATS/IDSA statement: diagnosis, treatment, and prevention of nontuberculous mycobacterial diseases. American journal of respiratory and critical care medicine, 175(4), 367416.

10-Halse, T. A., Escuyer, V. E., \& Musser, K. A. (2011). Evaluation of a Single Tube Multiplex Real-Time PCR for Differentiation of the Mycobacterium tuberculosis Complex in Clinical Specimens. Journal of clinical microbiology, JCM-00467.

11-Henkle, E., Hedberg, K., Schafer, S. D., \& Winthrop, $\quad K . \quad L$. (2017). Surveillance of ExtrapulmonaryNontuberculous

Mycobacteria Infections, Oregon, USA, 2007-2012. Emerging

infectious diseases, 23(10), 1627.

12-Henkle, E., Hedberg, K., Schafer, S. D., \& Winthrop, $\quad K . \quad L$. (2017). Surveillance of ExtrapulmonaryNontuberculous Mycobacteria Infections, Oregon, USA, 2007-2012. Emerging

infectious diseases, 23(10), 1627.

13- Hibah, N. A. A. (2015). Extrapulmonary tuberculosis situation in El-Behira 
Governorate, Egypt. Egyptian Journal of

Bronchology, 9(1), 96-100.

14- Huang, C. T., Chen, C. Y., Chen, H. Y., Chou, C. H., Ruan, S. Y., Lai, C. C., \&Hsueh, P. R. (2010). Genitourinary infections caused by nontuberculous mycobacteria at a university hospital in Taiwan,

1996-2008. Clinical Microbiology and Infection, 16(10), 1585-1590.

15- Khan, R., Abid, S., Jafri, W., Abbas, Z., Hameed, K., \& Ahmad, Z. (2006). Diagnostic dilemma of abdominal tuberculosis in non-HIV patients: an ongoing challenge for physicians. World journal of gastroenterology: WJG, 12(39), 6371.

16- Krishnaswamy, J., Rahaman, K., Reshma, S., \&Bharath, N. (2017). Abdominal tuberculosis: a clinical study in a tertiary care hospital. International Surgery Journal, 4(10), 3423-3426.

17- $m$ Kumar $N$ and Aggarwal R.(2003). Abdominal tuberculosis. In: API Textbook of Medicine, 7th ed. National Book Depot (Distributor): Mumbai. 562.

18- Kumari, R., Sinha, P., Banerjee, T., \&Anupurba, $S$. (2016).Isolation and characterization of Mycobacterium tuberculosis from extra pulmonary specimens in a tertiary referral hospital of north India. Journal of Advances in Medicine, 5(2), 25-28.

19- Makharia, G. K., Srivastava, S., Das, P., Goswami, P., Singh, U., Tripathi, M., ...\& Gupta, S. D. (2010).Clinical, endoscopic, and histological differentiations between Crohn's disease and intestinal tuberculosis. The American journal of gastroenterology, 105(3), 642.

20-Maurya, A. K., Nag, V. L., Kant, S., Kushwaha, R. A. S., Kumar, M., Singh, A. K., \& Dhole, T. N. (2015). Prevalence of nontuberculous mycobacteria among extrapulmonary tuberculosis cases in tertiary care centers in Northern
India. BioMed

research

international, 2015.

21-Mohammadien, H., Alkhayat, K., Hamed, A., \&Shaaban, M. (2017).Patterns, trends and treatment outcomes of extra-pulmonary tuberculosis in Sohag, Upper Egypt. Egyptian Journal of Chest Diseases and Tuberculosis, 66(2), 313316.

22-Mukewar, S., Mukewar, S., Ravi, R., Prasad, A., \&Dua, K. S. (2012). Colon tuberculosis: endoscopic features and prospective endoscopic follow-up after anti-tuberculosis treatment. Clinical and translational gastroenterology, 3(10), e24.

23- Noertjojo, K., Tam, C., Chan, S., \& Chan-Yeung, M. M. (2002).Extrapulmonary and pulmonary tuberculosis in Hong Kong. The International Journal of Tuberculosis and Lung Disease, 6(10), 879-886.

24- Peto, H. M., Pratt, R. H., Harrington, T. A., LoBue, P. A., \& Armstrong, L. R. (2009).Epidemiology of extrapulmonary tuberculosis in the United States, 19932006. Clinical Infectious Diseases, 49(9), 1350-1357.

25-Prakasha, S. R., Suresh, G., D'sa, I. P., Shetty, S. S., \& Kumar, S. G. (2013). Mapping the pattern and trends of extrapulmonary tuberculosis. Journal of global infectious diseases, 5(2), 54-59.

26- Rathi, P., \&Gambhire, $\quad P$. (2016).Abdominal tuberculosis. J Assoc Physicians India, 64(2), 38-47.

27- Roberto M.S., Adalgiza da Silva R., Flábio R.A., Antônio A. F., Andrea P., Philip N. S., Ronaldo R., Maria A. S. M., Mark D.C.G. (2018) Risk factors for human Mycobacterium bovisinfections in an urban area of Brazil, MemInstOswaldo Cruz, Rio de Janeiro, Vol. 113(8): e170445

28-Sadeghian,A., Tavakkol A., Ghanaat J., Sadeghain H., Namaii M. and Khodadoost M.(2005). Comparison of Three Methods for the Identification of 
Mycobacterium tuberculosis, INTERNATIONAL JOURNAL OF AGRICULTURE \& BIOLOGY15608530/07-2-203-206

29- Sharma, M. P., \& Bhatia, $V$. (2004). Abdominal tuberculosis. Indian Journal of Medical Research, 120, 305315.

30-Shenoy VP, Mukhopadhyay C.( 2014).Rapid Immunochromatographic Test for the Identification and Discrimination of Mycobacterium tuberculosis Complex Isolates from Nontuberculous Mycobacteria.J Clin DiagnRes. 2014;8(4):DC13-DC15.

31-Silaigwana, B., Green, E., \&Ndip, R. $N$. (2012). Molecular detection and drug resistance of Mycobacterium tuberculosis complex from cattle at a dairy farm in the Nkonkobe region of South Africa: A pilot study. International journal of environmental research and public health, 9(6), 2045-2056.

32- Singh, A. K., Dhole, T., Maurya, A. K., Kumar, M., Umrao, J., \& Nag, V. (2012). Evaluation of rapid TB antigen MPT64 test for identification of Mycobacterium tuberculosis complex in liquid culture isolates at tertiary care center in Northern India. International Journal of Infectious Diseases, 16, e294.

33-Sobh, E., Kinawy, S. A. E., Abdelkarim, Y. M. A., \&Arafa, M. A. (2016). The pattern of tuberculosis in Aswan Chest Hospital, Egypt. International journal of mycobacteriology, 5(3), 333-340. $\begin{array}{cccc}\text { 34- Somoskovi } & \text { A, Mester J, Hale } & \text { YM, } \\ \text { Parsons } & \text { LM, } & \text { Salfinger } & \text { M. } \\ \text { (2002).Laboratory } & \text { diagnosis } & \text { of }\end{array}$ nontuberculous mycobacteria. Clin Chest Med 2002;23:585-97.

35- Sreeramareddy, C. T., Panduru, K. V., Verma, S. C., Joshi, H. S., \& Bates, M. $N$. (2008). Comparison of pulmonary and extrapulmonary tuberculosis in Nepal-a hospital-based retrospective study. $B M C$ infectious diseases, 8(1), 8.

36- Tortoli, E., Bartoloni, A., Böttger, E. C., Emler, S., Garzelli, C., Magliano, E., ...\&Urbano, $P$. (2001).Burden of unidentifiable mycobacteria in a reference laboratory. Journal of clinical microbiology, 39(11), 4058-4065.

37- Umrao, J., Singh, D., Zia, A., Saxena, S., Sarsaiya, S., Singh, S., ...\& Dhole, T. $N$. (2016). Prevalence and species spectrum of both pulmonary and extrapulmonarynontuberculous mycobacteria isolates at a tertiary care center. International journal of mycobacteriology, 5(3), 288-293.

38- WHO (2014). Tuberculosis. Fact sheet no. 104. Updated March 2014

WHO,( 2017).Global Tuberculosis Report.

39- World Health Organization (2017). Global Tuberculosis

Report.WHO, Geneva, Switzerland.

40- Yang, Z., Kong, Y., Wilson, F., Foxman, B., Fowler, A. H., Marrs, C. F., ...\& Bates, J. H. (2004).Identification of risk factors for extrapulmonary tuberculosis. Clinical infectious diseases, 38(2), 199-205. 
